\title{
Archéopages
}

Archéopages

Archéologie et société

40 | 04-07/2014

Villages

\section{Un exemple d'habitat groupé au début du haut Moyen Âge. Le site de Warmeriville « La Bassière » (Marne)}

Vincent Marchaisseau et Gaëlle Pertuisot

\section{OpenEdition}

Journals

Édition électronique

URL : https://journals.openedition.org/archeopages/636

DOI : 10.4000/archeopages.636

ISSN : 2269-9872

Éditeur

INRAP - Institut national de recherches archéologiques préventives

Édition imprimée

Date de publication : 1 juin 2015

Pagination : 124-131

ISSN : 1622-8545

\section{Référence électronique}

Vincent Marchaisseau et Gaëlle Pertuisot, « Un exemple d'habitat groupé au début du haut Moyen Âge. Le site de Warmeriville «La Bassière » (Marne) », Archéopages [En ligne], 40 | 04-07/2014, mis en ligne le 01 juillet 2016, consulté le 21 janvier 2022. URL : http://journals.openedition.org/archeopages/ 636 ; DOI : https://doi.org/10.4000/archeopages.636 


\title{
Un exemple d'habitat groupé au début du haut Moyen Âge Le site de Warmeriville « La Bassière » (Marne)
}

\author{
Vincent Marchaisseau Inrap, UMR 6298, «Archéologie, terre, histoire, sociétes " \\ Gaëlle Pertuisot Inrap,
}

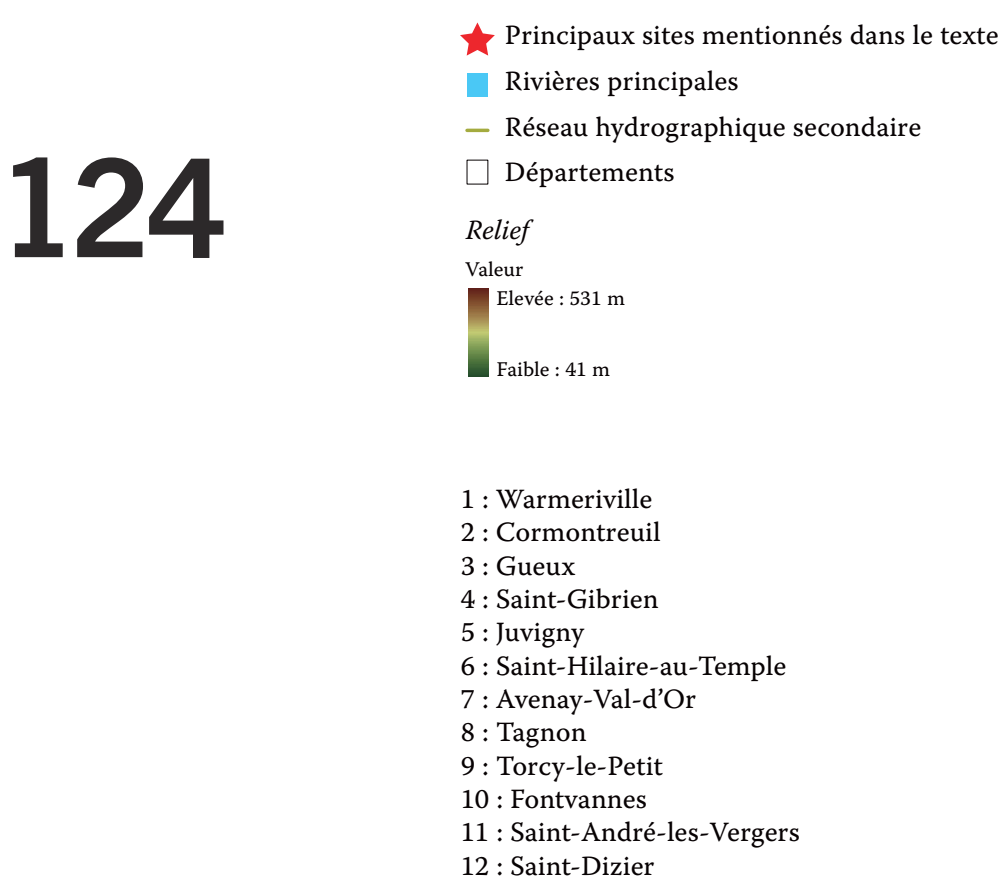

Si la notion de «village» est solidement ancrée dans l'imaginaire national, elle renvoie surtout au village du second Moyen Âge et de l'Ancien Régime: un habitat groupé, spécialement dans le paysage d'openfield de la Champagne crayeuse, qui comporte une église paroissiale et son cimetière, parfois un château (Beague-Tahon, Georges-Leroy, 1995; Pichot, 2002). Ce modèle idéal ne correspond qu'à des occupations rurales dont la mise en place définitive n'intervient pas avant les $\mathrm{XI}^{\mathrm{e}}-\mathrm{XII}^{\mathrm{e}}$ siècles, bien que leurs racines puissent remonter au $\mathrm{IX}^{\mathrm{e}}$ siècle (Bourdin, Durand, 200o; Gérard, 2012). L'historiographie a longtemps dénié à l'époque mérovingienne le terme de village (Pichot, 2002,

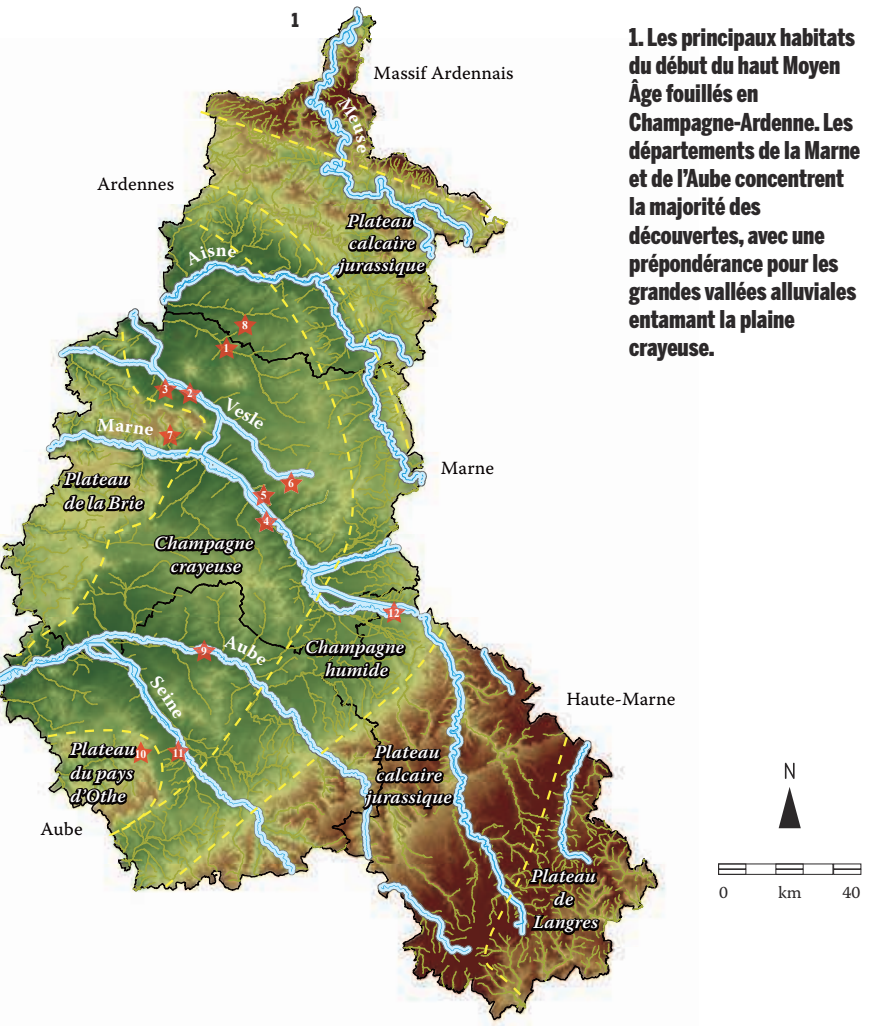

p. 6o-91) et les archéologues doivent concéder que l'« on peut douter de notre capacité à identifier archéologiquement un vicus du haut Moyen Âge, en dehors des cas où il prend la suite d'une agglomération secondaire antique [...]. On a tout autant de difficultés à identifier une villa du haut Moyen Âge» (Zadora-Rio, 2008, p. 72). Toutefois, des publications récentes font état d'une communauté villageoise sans doute dès le début du haut Moyen Âge, mais dont les formes de l'habitat sont distinctes du village «classique» qui émergera à la période suivante: on parle alors de «village faible» (Wickham, 2010; Nissen-Jaubert, 1998). 
2.Vue aérienne du secteur décapé en 2012. La zone humide actuelle se trouve au nord-est, sous les taillis; d'anciennes

dépressions humides sont parfaitement

visibles sous la forme de langues de limon grisâtre surligné d'un niveau organique noir. Ces niveaux étaient déjà asséchés durant le haut Moyen Âge, puisque une partie de la voie et de nombreuses structures domestiques s'y implantent.

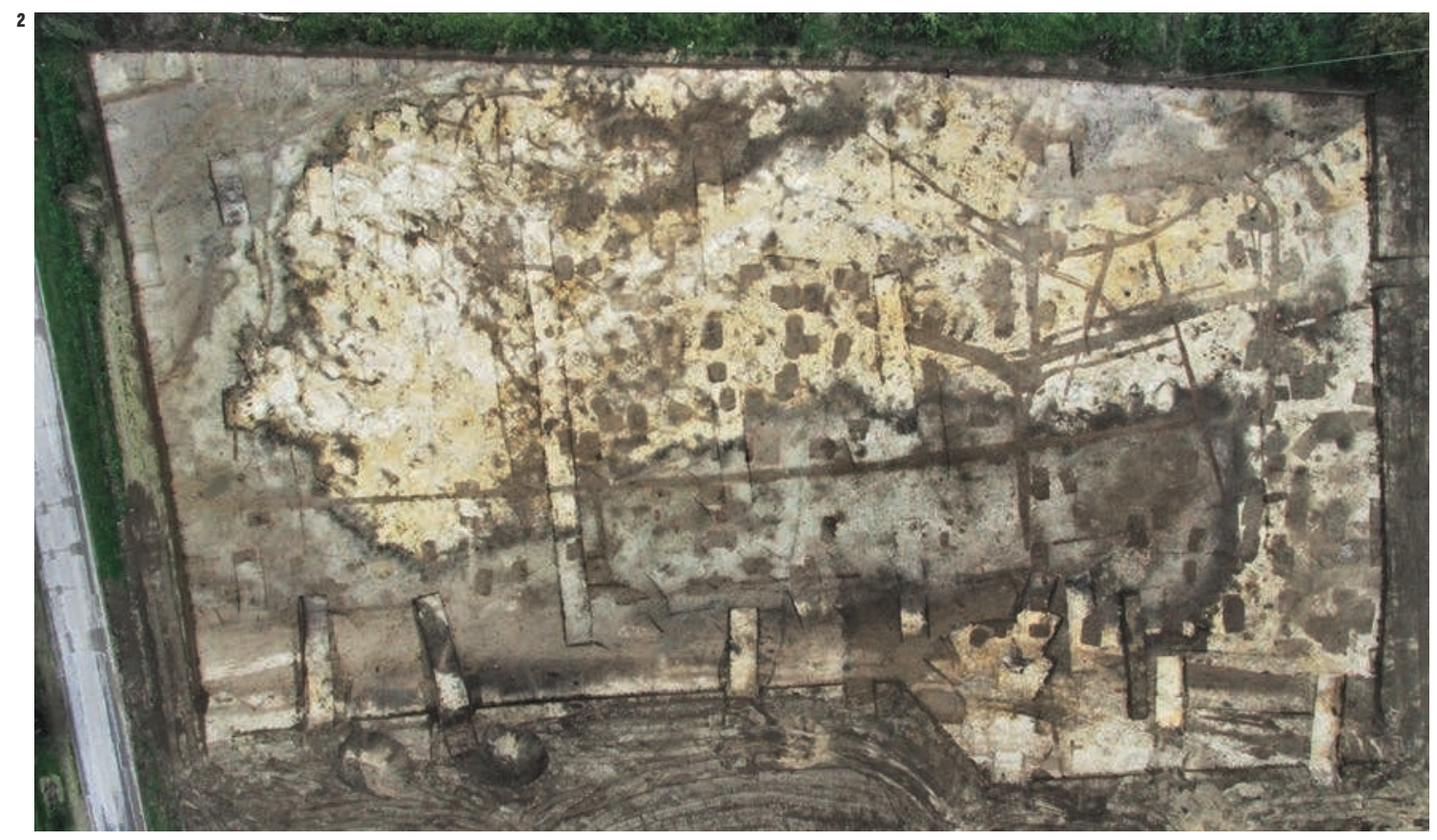

เ્ઞ

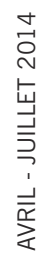

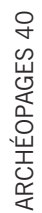

Limon argileux grisâtre/niveau organique

Craie

Structures archéologiques

Éléments du diagnostic (Rabasté 2010)

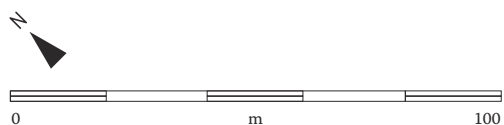




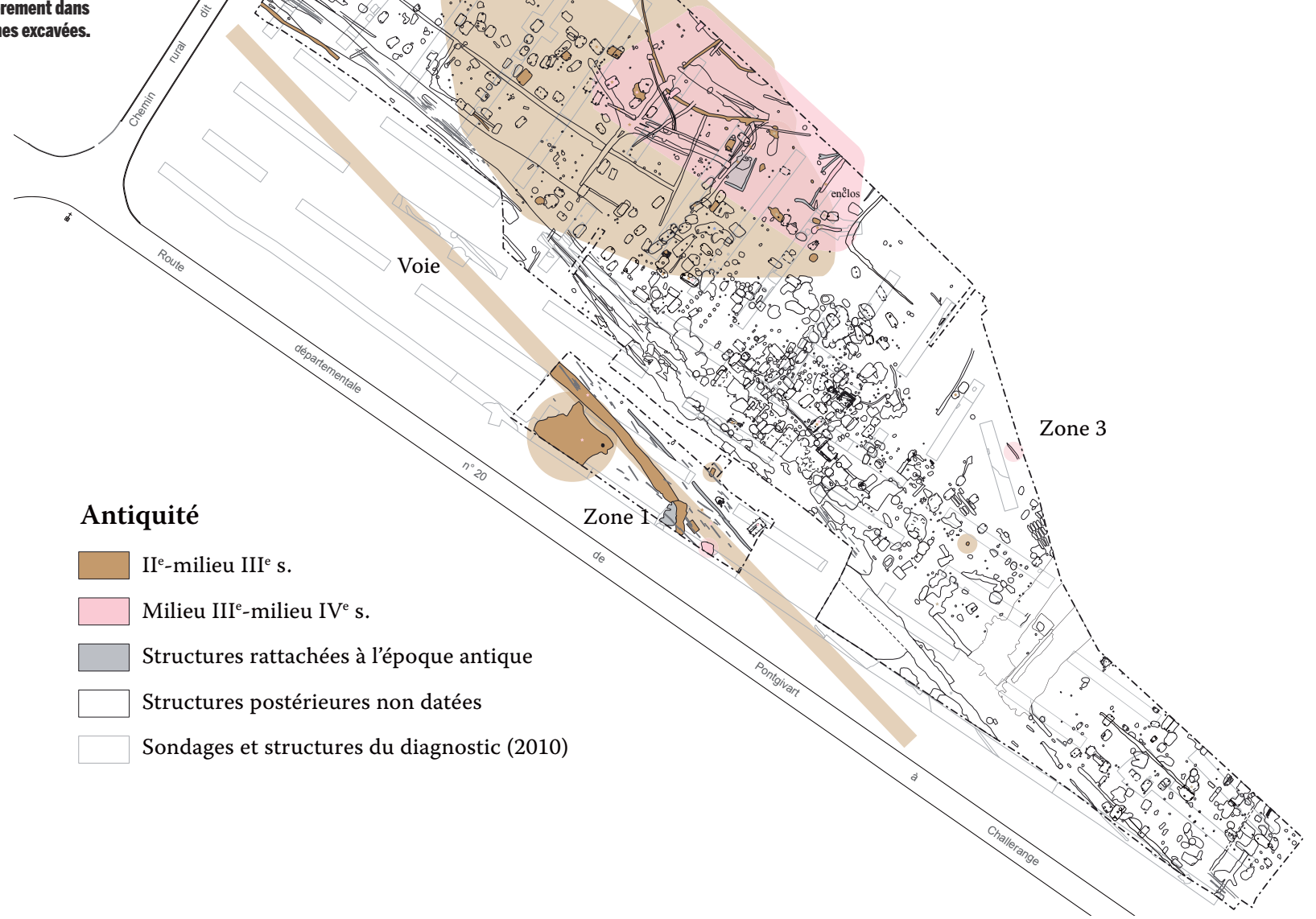

L'habitat rural de la transition Antiquité tardive/haut Moyen Âge n'est pas toujours aisé à percevoir en Champagne et, lorsqu'il est identifié, il se résume à quelques structures dont l'organisation est bien difficile à déterminer, car souvent masquée par les vestiges d'autres époques [ill. 1]. La découverte d'un habitat dense du haut Moyen Âge sur la commune de Warmeriville, au lieu-dit «La Bassière», en 2012-2013 (superficie 2,1 ha), peut constituer un jalon dans la reconnaissance des habitats groupés des $\mathrm{V}^{\mathrm{e}}$-VIII ${ }^{\mathrm{e}}$ siècles.

\section{L'environnement et les occupations antiques}

La commune de Warmeriville se situe à $20 \mathrm{~km}$ au nord-ouest de la ville de Reims, dans la vallée de la Suippe, affluent de l'Aisne. Cette zone, densément explorée par l'archéologie préventive depuis une quinzaine d'années, témoigne d'une densification de l'occupation humaine dès le Bronze final. L'époque antique est particulièrement bien représentée, bénéficiant de la proximité d'une capitale provinciale depuis laquelle se développe un réseau routier d'intérêt suprarégional. Localement, le site de «La Bassière» se trouve sur la rive gauche de la Suippe, en bordure d'une zone humide représentée par d'anciennes dépressions comblées d'un limon argileux gris surmontant un niveau organique noirâtre asséché, voire encore humide dans la partie basse du site [ill. 2]. Au centre de l'emprise, un niveau de craie pouvant atteindre $1 \mathrm{~m}$ d'épaisseur accueille la densité maximale 


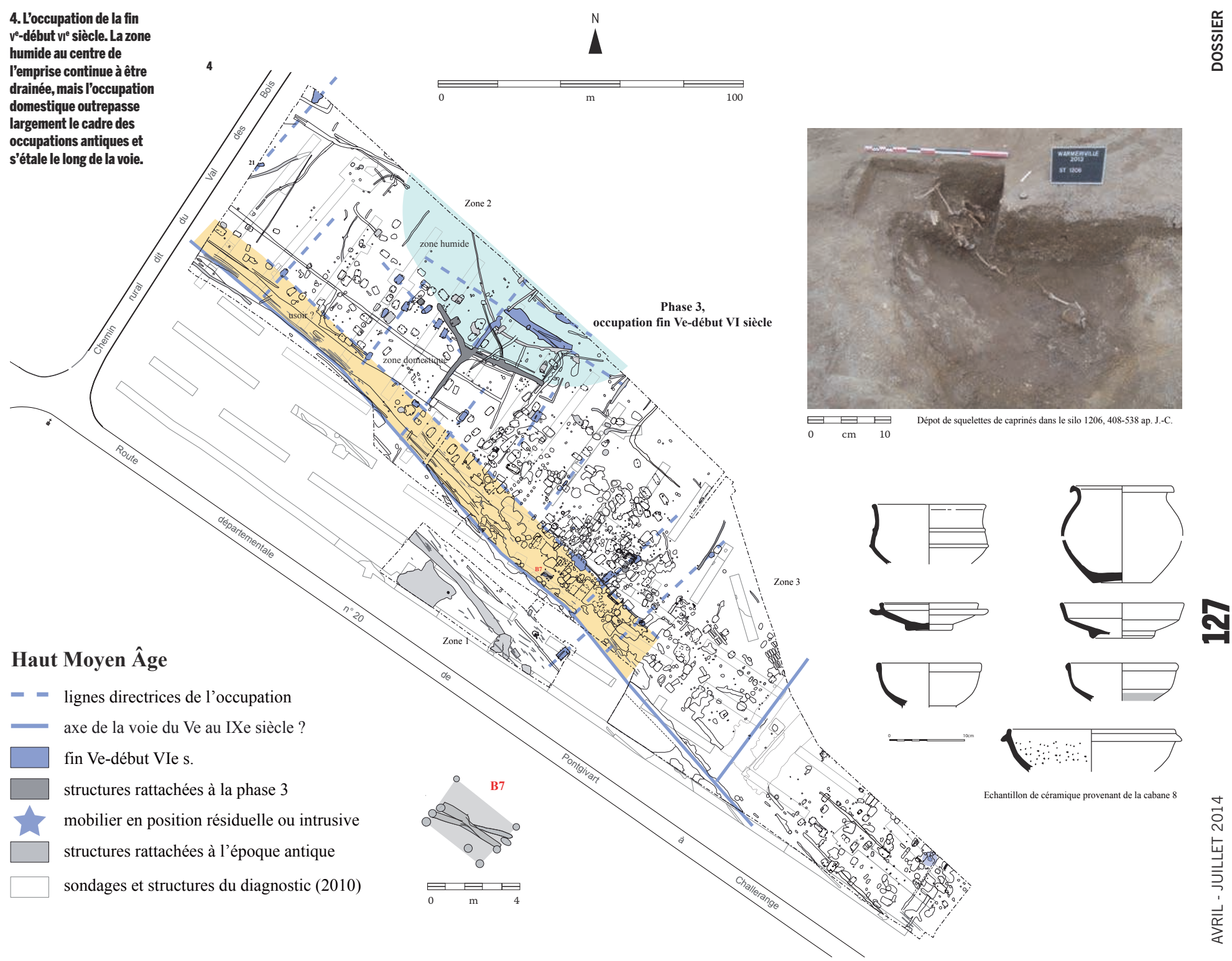

de vestiges. À $45 \mathrm{~m}$ environ vers le sud-ouest, un axe antique, sous la forme d'un chemin creux, traverse une partie de l'emprise. Cet axe viaire constitue le vestige le plus ancien reconnu et l'élément ordonnateur des occupations postérieures. En effet, toutes les occupations domestiques vont par la suite se limiter à cette bande étroite entre voie et zone humide, ce qu'attestent les données des diagnostics sur les parcelles attenantes (Rabasté, 2008, 2010).

L'occupation antique recouvre la fin du HautEmpire et le début de l'Antiquité tardive ( $\mathrm{II}^{\mathrm{e}}$-milieu $\mathrm{IV}^{\mathrm{e}}$ siècle); elle se résume à quelques vestiges épars, même si une concentration au plus près de la zone humide avec les premières tentatives de drainage est perceptible [ill. 3]. Un seul bâtiment sur fondations de craie a été repéré, il est alors envisageable que l'habitat en lui-même soit situé en dehors de l'emprise. À proximité de la zone humide, quelques fossés dessinent un enclos avec une entrée en chicane qui peut être assimilé à un enclos de pâturage ou de regroupement du bétail. Aucune structure domestique n'a pu être datée entre le milieu du IV ${ }^{\mathrm{e}}$ et le milieu du $\mathrm{v}^{\mathrm{e}}$ siècle, constituant ainsi un hiatus dans l'occupation. Ce hiatus ne touche vraisemblablement que l'habitat, tandis que le terroir continue à être exploité.

\section{L'occupation de la fin du $\mathbf{v}^{\mathrm{e}}$-début du vi $\mathrm{I}^{\mathrm{e}}$ siècle}

D'après les trouvailles monétaires, la voie romaine semble définitivement abandonnée vers la fin $\mathrm{du} \mathrm{IV}^{\mathrm{e}}$ siècle, mais un autre axe la relaie; bien 
que décalée désormais de 10 à 20 m vers le nordest, cette nouvelle voie conserve la même orientation. Dès cette période, l'occupation paraît s'étaler le long de la voie et ne se contente plus uniquement des abords de la zone humide [ill. 4]. Celle-ci continue néanmoins à être drainée par l'ajout d'au moins deux fossés dont l'un, par son ampleur, peut être qualifié de fossé-collecteur. Cette phase voit l'apparition des premières cabanes excavées; plusieurs comprennent quatre poteaux d'angle et un poteau supplémentaire au milieu des petits côtés. Une cabane possède un aménagement particulier: deux solins, constitués de blocs de grès et de tuiles, disposés en vis-à-vis le long des grands côtés de l'excavation; la charpente est soutenue par une file de trois poteaux centraux.

Un alignement de structures (cabanes excavées, fossés linéaires, fosse d'extraction, silo) suit l'orientation de la voie, entre 8 et $12 \mathrm{~m}$ de distance; cet alignement paraît ménager un espace vide entre la zone domestique et l'axe viaire. Seul un petit bâtiment sur poteaux est implanté dans ce secteur (B7); deux rigoles disposées en X en son centre ont livré des taux de phosphore compatibles avec une fonction de stabulation. Les autres alignements de structures perceptibles sont disposés perpendiculairement à la voie, tandis que deux zones de concentration de vestiges apparaissent. Une cabane se situe sur le bord sudouest du chemin creux (cabane 8); il s'agit de la seule structure ayant servi de dépotoir domestique, qui représente à elle seule le quart du mobilier céramique de cette phase.

Un assemblage faunique singulier a été découvert dans un silo: plusieurs carcasses incomplètes de caprinés (932 restes) ont été déposées sans ménagement dans la partie médiane de la fosse de stockage déjà en grande partie comblée, témoignant d'un épisode d'épizootie. Une datation radiocarbone a livré un intervalle calibré similaire à la datation d'une graine carbonisée provenant d'une rigole du bâtiment sur poteaux. Léconomie est clairement tournée vers l'agriculture et l'élevage semble tenir une place importante.

\section{L'occupation des VI $^{\mathrm{e}}$-VIII ${ }^{\mathrm{e}}$ siècles}

Dès la période suivante, l'occupation s'intensifie [ill. 5]. Un troisième et un quatrième pôle de concentration de structures font leur apparition. Ces zones regroupent essentiellement des cabanes excavées, 80 d'entre elles pouvant se rapporter aux $\mathrm{VI}^{\mathrm{e}}$-VIII ${ }^{\mathrm{e}}$ siècles.

Au sud-est de l'emprise, quelques segments de fossés délimitent une bande vide de structure entre ces derniers et l'axe viaire (fossés bordiers); cette limite se poursuit ensuite par un alignement de vestiges comme lors de la phase précédente. Cette limite parallèle à la voie offre l'image des « usoirs » typiques des villages-rue des $\mathrm{IX}^{\mathrm{e}}$-XII ${ }^{\mathrm{e}}$ siècles (Gérard, 2012, p. 41); cette acception est sans doute peut-être anachronique pour les $\mathrm{VI}^{\mathrm{e}}$-VIII ${ }^{\mathrm{e}}$ siècles, mais il existe de toute évidence un certain respect des distances par rapport à la bande de roulement du chemin, dans la continuité de la période précédente. L'occupation domestique de Warmeriville se cantonne par ailleurs à un unique côté de la voie: seuls une fosse, une sépulture et un four outrepassent l'axe viaire et se trouvent «isolés » sur son côté sud-ouest.

La zone drainée antérieurement par des fossés paraît toujours en fonction, même si les datations proviennent désormais des structures alentour et non plus des fossés eux-mêmes.

Stratigraphiquement, deux phases ont pu être définies avec un changement dans la zone d'exutoire. Au plus près de la zone humide, deux enclos fossoyés ont été repérés. Les systèmes d'ouverture et de division de l'espace plaident en faveur de sas répartiteurs et donc d'enclos de pâturage. L'enclos sud-ouest pourrait reprendre en partie le fossé d'un enclos de l'Antiquité tardive, peut-être significatif d'une continuité de l'occupation et des fonctions de ce secteur agricole. Ces structures fossoyées, ainsi que quelques cabanes, forment une ligne approximativement parallèle à la voie. Le secteur domestique se situe entre ces deux limites, sur une largeur réduite à moins de $40 \mathrm{~m}$. Les deux noyaux de structures de la fin $\mathrm{V}^{\mathrm{e}}$-début du $\mathrm{VI}^{\mathrm{e}}$ siècle sont toujours occupés et se densifient. Des alignements perpendiculaires à la voie sont désormais lisibles sur la totalité de la largeur de l'espace domestique. Au sud-ouest d'un fossé de drainage se trouve une nouvelle concentration de cabanes, la plus importante de cette phase et associée à un bâtiment sur poteaux. Une zone dédiée à l'extraction existe à proximité d'un chemin creux se dirigeant vers le cour de la zone humide. Au sud-ouest de l'opération se dessine un nouveau noyau de structures, en liaison semble-t-il avec des fossés de parcellaire.

Seuls deux bâtiments de plain-pied sur poteaux ont pu être rattachés à cette période. Le bâtiment 6 , de plan régulier à deux nefs $\left(38 \mathrm{~m}^{2}\right)$, résiste à l'interprétation : petit bâtiment d'habitation ou bâtiment agricole de stockage ? L'exiguïté de ces bâtiments, quelle que soit leur fonction, semble une caractéristique de ce secteur du nord de la Champagne et des Ardennes (Billoin, 1998; Truc, 2005; Flotté, 2009). Le bâtiment 2 est quant à lui clairement une annexe agricole (moins de $10 \mathrm{~m}^{2}$ ). On peut donc de nouveau s'interroger sur la fonction d'habitat de cette phase. Pourrions-nous être en présence uniquement d'un secteur dévolu à l'agriculture? Le mobilier, bien qu'indigent, la diversité des structures (cabanes, silos, puits, fosses d'extraction...) et leur organisation raisonnée, plaident néanmoins en faveur d'un habitat dont les principaux bâtiments seraient à rechercher dans les noyaux de trous de poteau non datés et en grande partie bouleversés par les occupations postérieures. Si l'on prend en compte les orientations des différents éléments structurants (voie, fossés bordiers, alignements de 
Haut Moyen Âge

- - Lignes directrices de l'occupation

Axe de la voie du $\mathrm{V}^{\mathrm{e}}$ au $\mathrm{IX}^{\mathrm{e}}$ siècle? $\mathrm{VI}^{\mathrm{e}}-\mathrm{VIII}^{\mathrm{e}} \mathrm{s}$.

Structures rattachées à la phase 4

Fin VIII', IXes.

Structures rattachées à la phase 3 (fin $V^{\mathrm{e}}$-début VI ${ }^{\mathrm{e}} \mathrm{s}$.)

Sondages et structures du diagnostic (2010)

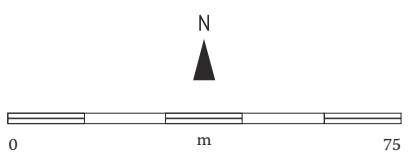

Phase 4,

occupation VIe-VIIIe siècles
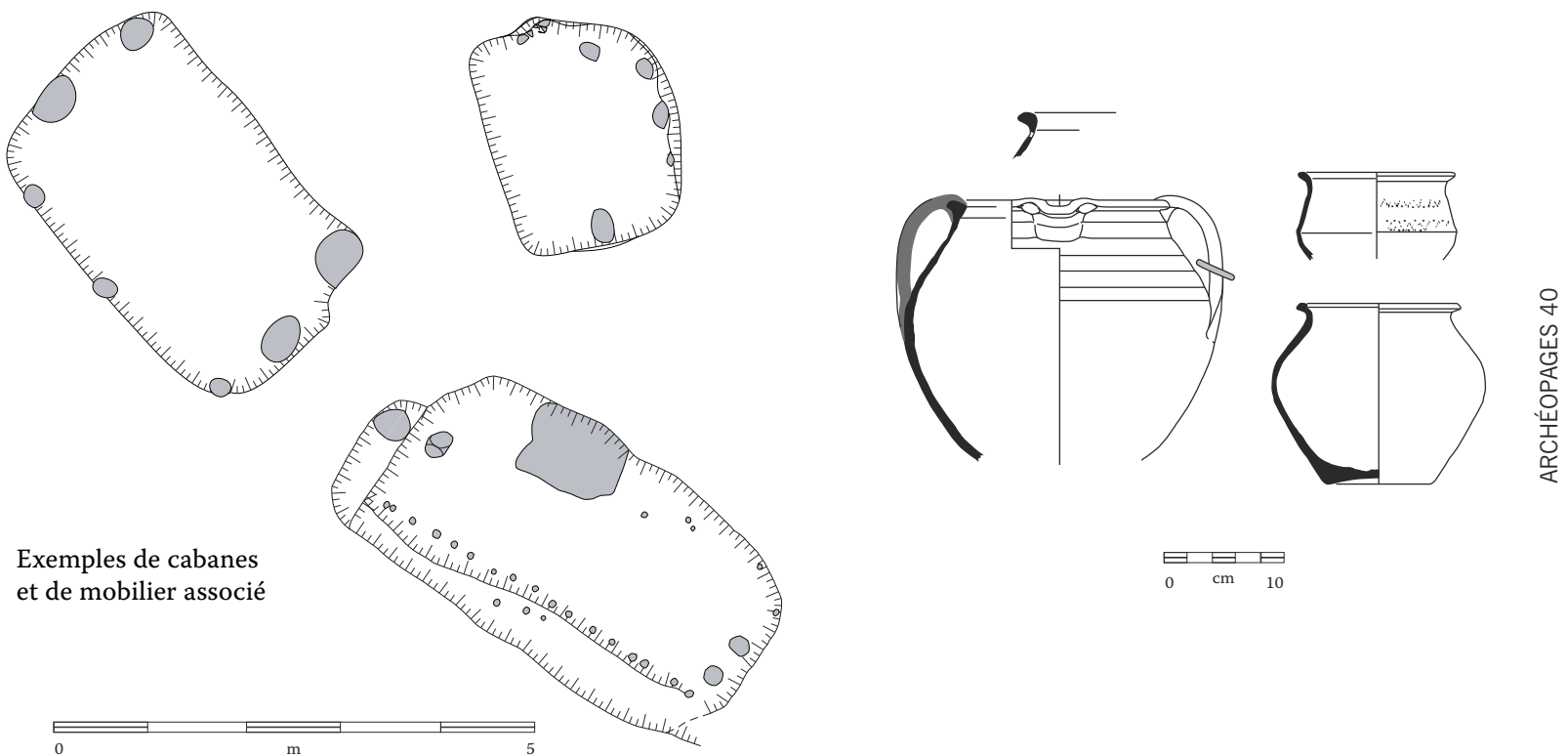
vestiges...), on aboutit à une organisation rationnelle où les divergences d'axes sont mineures; en moyenne, les alignements de structures domestiques sont quasi perpendiculaires à l'axe viaire avec une orientation à $87,89^{\circ}$. Cette organisation et le nombre de structures recensées (environ 150) autorisent selon nous à parler ici d'habitat groupé.

Les deux premières sépultures, situées à proximité de la voie et de même orientation, font leur apparition durant la seconde moitié du $\mathrm{VII}^{\mathrm{e}}$ siècle. Sur les neuf sépultures recensées, la plupart appartiennent toutefois à la phase suivante (fin $\mathrm{VIII}^{\mathrm{e}}-\mathrm{IX}^{\mathrm{e}}$ siècle). L'économie du site est toujours dédiée à la production agricole et notamment à l'élevage au cours des $\mathrm{VI}^{\mathrm{e}}$-VIII ${ }^{\mathrm{e}}$ siècles. Aucune activité artisanale n'a pu être détectée et aucune hiérarchisation dans les secteurs domestiques ne se fait jour. Le mobilier céramique lui-même présente un aspect surprenant pour la période (étude N. Mahé-Hourlier, Inrap CIF) : on remarque la suprématie des pots à cuire, et notamment des marmites à suspension, sur toutes les autres catégories; il faut souligner l'indigence des formes ouvertes qui ne totalisent que 5,3\% des individus identifiés et ne sont souvent pas représentées par plus d'un tesson (NMI/NR $=0,8)$.

L'appauvrissement du vaisselier aux seules formes fermées n'est généralement pas discernable avant le $\mathrm{IX}^{\mathrm{e}}$ siècle. Nous ne disposons malheureusement d'aucun ensemble de comparaison à l'heure actuelle pour savoir si ce déséquilibre est induit par le vaisselier de ce secteur ou s'il s'agit d'un choix en relation avec les activités pratiquées au sein des cabanes excavées, principales structures pourvoyeuses de mobilier.

\section{Les occupations des $\mathrm{IX}^{\mathrm{e}}-\mathrm{XII}^{\mathrm{e}}$ siècles}

À partir de la fin du VIII et durant tout le $\mathrm{IX}^{\mathrm{e}}$ siècle, l'occupation se réorganise. Quelques vestiges épars se rencontrent encore le long de l'axe viaire, mais l'essentiel de l'habitat se concentre maintenant autour des deux plus importants pôles de la période antérieure, entourés de nombreuses fosses d'extraction de gravier et de limon crayeux. On peine toujours à définir des bâtiments d'habitation, mais l'occupation est désormais centrée autour d'un espace central vide, sans doute une cour. Après un hiatus difficilement interprétable dans le courant $\mathrm{du} \mathrm{x}^{\mathrm{e}}$ siècle selon l'étude céramique, l'occupation du XI ${ }^{\mathrm{e}}$-début du $\mathrm{XII}^{\mathrm{e}}$ siècle se trouve regroupée presque entièrement sur moins de $2000 \mathrm{~m}^{2}$. Il est délicat de parler encore d'habitat groupé, malgré la présence de trois bâtiments construits sur solins, un seul ayant une superficie compatible avec une habitation $\left(54 \mathrm{~m}^{2}\right)$. Ces deux dernières phases d'occupation domestique du site sont plus classiques dans leur organisation spatiale et trouvent de nombreux parallèles tant en Champagne que dans l'ensemble du nord de la France.

\section{La spécificité de l'habitat de Warmeriville au début du haut Moyen Âge}

Plus de 80 sites d'habitat du haut Moyen Âge sont repérés en Champagne-Ardenne; nous devons cependant constater un déficit documentaire pour la période des $\mathrm{V}^{\mathrm{e}}$-VIII ${ }^{\mathrm{e}}$ siècles. Des habitats denses sont connus, mais uniquement à partir de la fin du viII ${ }^{\mathrm{e}}$ et surtout du IX ${ }^{\mathrm{e}}$ siècle: on peut citer, dans le département de la Marne, Cormontreuil (Rabasté, à paraître), Avenay-Val-d'Or (Verbrugghe, à paraître), Juvigny (Beague-Tahon, Georges-Leroy, 1995), Gueux (Koehler, 200o) et Saint-Gibrien (Villes, 1995); dans les Ardennes, Tagnon (Truc, 2005); dans l'Aube, Torcy-le-Petit (Beague-Tahon, GeorgesLeroy, 1995) et Fontvannes (Gourgousse, 1992); enfin, en Haute-Marne, Saint-Dizier (BeagueTahon, 1993). Parmi ces occupations, Fontvannes est le seul habitat de type village-rue présentant des usoirs et une organisation en parcelles laniérées datant des $\mathrm{IX}^{\mathrm{e}}-\mathrm{XI}^{\mathrm{e}}$ siècles.

Il apparaît que les sites marnais sont généralement pourvus de très nombreuses cabanes excavées (30o à Saint-Gibrien, environ 120 à Avenay-Val-d'Or, 100 à Gueux, 83 à Cormontreuil) tandis que l'on ne dépasse pas la trentaine de vestiges de ce type sur les sites aubois, à l'exception notable de Torcy-le-Petit (109). À cet égard, Warmeriville s'intègre au corpus marnais avec 228 cabanes recensées toutes périodes confondues. La particularité de ce site réside davantage dans sa chronologie précoce: aucune des occupations champenoises des $\mathrm{VI}^{\mathrm{e}}$-VIII ${ }^{\mathrm{e}}$ siècles n'a livré jusqu'à présent autant de structures possédant de surcroît une organisation aussi rigoureuse, planifiée, serait-on tenté de dire. En effet, la division de l'espace entre un secteur public (le chemin creux et l'usoir), une zone domestique (habitat privé, unités d'exploitation juxtaposées) et une zone agricole suggère une gestion raisonnée de l'espace au sein d'un habitat groupé. Nous sommes en présence d'une organisation linéaire «rectilinear settlements » d'après Hamerow, 2012, p. 73-83) préfigurant le village-rue carolingien et du Moyen Âge central. Cette distribution de l'habitat n'a toutefois pas connu de postérité à « $\mathrm{La}$ Bassière ». La plupart des occupations reconnues entre la fin du v $\mathrm{v}^{\mathrm{e}}$ et le viI ${ }^{\mathrm{e}}$ siècle dans le nord de la France sont généralement qualifiées de diffuses (Peytremann, 2003, vol. 1, p. 318-322; MahéHourlier, Poignant, 2012). En dehors des habitats de hauteur du monde méditerranéen ou du sud de l'Allemagne, les meilleures comparaisons d'habitats groupés en contexte rural semblent se retrouver à Mondeville dans le Calvados (Carpentier, Hincker, 2013), Genlis en Côte-d'Or (Catteddu, 1992) ou encore Mucking en Angleterre (Hamerow, 2012, p. 67-72).

Si la cohérence du plan de Warmeriville doit résulter d'un contrôle par une autorité supérieure, alors il convient de s'interroger sur le rôle des institutions religieuses dans la constitution et la 
structuration des habitats ruraux (Verslype, 2010, p. 241-242). Le rôle apparaît prépondérant dans les diocèses de Reims et Châlons, ce que confirment les sources textuelles: les villages de Bazancourt, Isle-sur-Suippe et Warmeriville, contigus, appartenaient en partie au domaine de l'abbaye Saint-Remi de Reims. Cependant, les archevêques « sont sans doute à l'origine de la donation de Bazancourt [...] et il en va de même pour le domaine d'Isle-sur-Suippe, dépendance de l'episcopium rémois» (Devroey, 1984, p. XCIX et CI). Le même processus a pu se produire pour Warmeriville qui apparait sous la forme Vuarmerii Villa dans une liste des dîmes de Saint-Timothée (dernier quart du $\mathrm{x}^{\mathrm{e}}$ siècle) et dans la première liste de cens de Saint-Remi (première moitié du XI ${ }^{\mathrm{e}}$ siècle; ibid., p. 90 et 110). Par ailleurs, de véritables «entreprises de peuplement» à l'initiative des archevêques rémois sont connues depuis le début du viI ${ }^{\mathrm{e}}$ siècle grâce à Flodoard et son Histoire de l'église de Reims (Devroey, 1990 et 2006, p. 85-87). Se poserait alors la question du statut des habitats: libres ou non libres ? Le peu d'éléments en notre possession invite à la prudence, mais l'hypothèse mérite réflexion.

La rapidité du développement économique actuel de ce secteur de la vallée de la Suippe laisse peut-être une chance d'appréhender prochainement la nécropole des occupants de «La Bassière», seul élément à même d'apporter de nouvelles informations sur le statut de ses habitants.

\section{Références bibliographiques}

BeAgUe-TAHON N., 1993, Rapport de fouilles archéologiques réalisées au centre d'activités commerciales et tertiaires du Chênes SaintAmand, lieu-dit «Le Seugnon-Toupot-Millot» à Saint-Dizier (Haute-Marne), Afan/Drac Champagne-Ardenne, $80 \mathrm{p}$.

Beague-Tahon N., Georges-Leroy M., 1995, «Deux habitats ruraux du haut Moyen Âge en Champagne crayeuse: Juvigny et Torcy-le-Petit (milieu VII ${ }^{\mathrm{e}}$-IX $\mathrm{e}^{\mathrm{e}}$ siècle) », in LORREN C., PERrIN P. (DIR.), L'Habitat rural du haut Moyen Âge (France, Pays-Bas, Danemark et Grande-Bretagne), Actes des XIVe Journées internationales d'archéologie mérovingienne, Guiry-en-Vexin / Paris, 4-8février 1993, Rouen, Association française d'archéologie mérovingienne (coll. Mémoires de l'Afam, 6), p. $175-183$.

Billoin D., 1998, «Un habitat du X $\mathrm{X}^{\mathrm{e}}$-XI ${ }^{\mathrm{e}}$ siècle à SousChâtillon, Boulzicourt RN 51 (Ardennes) ", Bulletin de la Société archéologique champenoise, t. 89, 2, p. 39-63.

Bourdin M., DURAND R., 200o, Vivre au village au Moyen Âge. Les solidarités paysannes du XI $I^{e}$ au $X I I I^{e}$ siècle, Rennes, Presses universitaires de Rennes, $207 \mathrm{p}$.

CATteddu I., 1992, «L'habitat rural mérovingien de Genlis (Côte-d'Or) », Revue archéologique de l'Est, 43, p. 39-98.

Carpentier V., Hincker V., 2013, «L'habitat rural du haut Moyen Âge en Basse-Normandie. Arrêté sur vingt années de recherches archéologiques ", in LORREN C. (DIR.), La Gaule, le monde insulaire et l'Europe du Nord au haut Moyen Âge. Actualité de l'archéologie en Normandie ( $\left.v^{e}-X^{e} s.\right)$, Actes des XXVII Journées internationales d'archéologie mérovingienne, Caen, 29 septembre-

$1^{\text {er }}$ octobre 2006, Rouen, Association française d'archéologie mérovingienne (coll. Mémoires de l'Afam, 28), p. 183-210.

Devroey J.-P., 1984, Le Polyptyque et les listes de cens de l'abbaye de Saint-Remi de Reims (IX ${ }^{e}-X I^{e}$ siècles), Reims, Académie nationale de Reims (coll. Travaux de l'Académie nationale de Reims, 163), 164 p.

DeVroey J.-P., 1990, «Les préoccupations de gestion des évêques de Reims (VI ${ }^{\mathrm{e}}$-IX $\mathrm{IX}^{\mathrm{e}}$ siècles) », in CLAUSE G., Guilbert S. et VAISSE M. (DIR.), $L a$ Champagne et ses administrations à travers le temps, Actes du colloque d'histoire régionale, Reims / Châlons-sur-Marne, 4-6 juin 1987, Lyon, La Manufacture (coll. L’Histoire partagée), p. 54-68.

DeVRoey J.-P., 2006, « Libres et non-libres sur les terres de Saint-Remi de Reims : la notice judiciaire de Courtisols (13 mai 847) et le polyptique d'Hincmar ", Journal des savants, 1, p. 65-103. Flotté D., 2009, «Le site médiéval de Saint-Hilaire-
au-Temple, "Le Raidon" (Marne) », in

VANMOERKERKe J. (DIR.), Le Bassin de la Vesle du Bronze final au Moyen Âge à travers les fouilles $d u$ TGV Est, Reims, Société archéologique champenoise (Bulletin de la Société archéologique champenoise, t. 102, 2), p. 306-334.

GÉRARD F., 2012, «Structuration du village pour une économie agraire planifiée à la fin du IX ${ }^{\mathrm{e}}$ siècle en Lorraine. Les sites de Vitry-sur-Orne et Demange aux-Eaux», Archéopages, 34, p. 38-47.

Gourgousse Y, 1992, Fontvannes "Les Tomelles». Une occupation médiévale des $\mathrm{IX}^{e}$-XI $\mathrm{I}^{e}$ siècles, Rapport de sauvetage urgent, Afan, Drac Champagne-Ardenne, $107 \mathrm{p}$.

Hamerow H., 2012: Rural Settlements and Society in Anglo-Saxon England, Medieval History and Archaeology, Oxford University Press, 194 p.

Koenler A., 200o, «Gueux, Les Terres brûlées», Bulletin scientifique régional ChampagneArdenne, p. 106-107.

NisSEN-JAUBERT A., 1998, « Habitats ruraux et communautés rurales", in Ruralia II, Colloque de Spa, Belgique, 1-7 septembre 1997, Prague, Institute of Archeology (coll. Památki archeologické, 11), p. 213-225.

Mahé-Hourlier N., Poignant S. (Dir.), 2012, Archéologie du village, archéologie dans le village dans le nord de la France du $v^{e}$ au XIII ${ }^{e}$ siècle, Actes de la table ronde du Musée d'archéologie nationale, Saint-Germain-en-Laye, 22-24 novembre 2007, Saint-Germain-en-Laye, Association française d'archéologie mérovingienne (coll. Mémoires de l'Afam, 29), $250 \mathrm{p}$.

Peytremann E., 2003, Archéologie de l'habitat rural du haut Moyen Âge dans le nord de la France du IV au XII ${ }^{e}$ siècle, Saint-Germain-en-Laye, Association française d'archéologie mérovingienne (coll. Mémoires de l'Afam, 23), 2 vol.

Ріснот D., 2002, Le Village éclaté. Habitat et société dans les campagnes de l'Ouest au Moyen Âge, Rennes, Presses universitaires de Rennes, $395 \mathrm{p}$.

RABASTÉ Y., 2008, Warmeriville, «La Bassière, parcelle $A B n^{\circ} 143$ ", Rapport de diagnostic archéologique, Inrap, Drac Champagne-Ardenne, $79 \mathrm{p}$.

RABASTÉ Y., 2010, Warmeriville, «La Bassière». Un habitat rural du haut Moyen Âge sur la commune de Warmeriville, Inrap, Drac ChampagneArdenne, $84 \mathrm{p}$

Truc M.-C., 2005, Tagnon «Les Cosserons» (Ardennes). Un habitat rural du haut Moyen Âge (VIII ${ }^{e}-X I^{e}$ siècles), Rapport de fouille archéologique préventive (mai-juin 2003), Inrap, Drac Champagne-Ardenne, 3 vol.

VERSLYPE L., 2010, «Avant le village, l'habitat rural mérovingien. Les sources archéologiques au nord-ouest des royaumes francs (fin $\mathrm{V}^{\mathrm{e}}$-VIII ${ }^{\mathrm{e}}$ siècles) », in YANTE J.-M., BultotVerleysen A.-M. (DIR.), Autour du «village». Établissements humains, finages et communautés rurales entre Seine et Rhin (IV $V^{e}-X I I I^{e}$ siècles), Actes du colloque international de Louvain-la-Neuve, 16-17 mai 2003, Louvain-la-Neuve, Institut d'études médiévales de l'Université catholique de Louvain, p. 237-275.

VILLES A., 1995, «Saint-Gibrien (Marne), au-dessus du Vieux-Pont. L'occupation du haut Moyen Âge», Les fouilles archéologiques sur le tracé de l'autoroute A 26 Châlons-sur-Marne/Troyes, Note préliminaire, sous la direction de A. Villes, p. 109-114

WICKHAM C., 2010, «L'identité villageoise entre Seine et Rhin, 500-80o ", in YAnte J.-M., BultotVERLEYSEN A.-M. (DIR.), Autour du «village». Établissements humains, finages et communautés rurales entre Seine et Rhin (IV $V^{e}-X I I I^{e}$ siècles), Actes du colloque international de Louvain-la-Neuve, 16-17 mai 2003, Louvain-la-Neuve, Institut d'études médiévales de l'Université catholique de Louvain, p. 141-151.

ZADORA-RIo É. (DIR.), 2008, Des paroisses de Touraine aux communes d'Indre-et-Loire. La formation des territoires, Tours, FERACF (coll. Suppl. à la RACF, 34), $303 \mathrm{p}$. 